\title{
Avaliação Imediata da Dor e Edema em Lesão Muscular Induzida por Formalina e Tratada com Laser $808 \mathrm{~nm}$
}

\section{Immediate Pain and Edema Assessment in Muscular Injury Induced by Formalin And Treated With Low-level Laser (808nm)}

\section{Emerson Borato}

Jaques Jean Junqueira Oliveira

Adriano Polican Ciena

Gladson Ricardo Flor Bertolini

Laboratório do Grupo de Estudo das Lesões e Recursos Fisioterapêuticos (GELRF) da Universidade Estadual do Oeste do Paraná (UNIOESTE), Cascavel - Paraná;

\section{Endereço para correspondência:}

Gladson Ricardo Flor Bertolini

Endereço Travessa Sebastião Pereira de Camargo, 69 - Jardim Padovani

- Cascavel - PR - CEP 85803-328.

E-mail: gladson_ricardo@yahoo.com.br

Submetido em 25/01/2007

Versão final recebida em 28/03/2008 Aceito em 11/04/2008

\begin{abstract}
RESUMO
O laser de baixa potência induz a bioestimulação celular, podendo acelerar a cicatrização de feridas, promover a regeneração do músculo esquelético, diminuir a resposta inflamatória, estimular a neoformação de vasos sanguíneos e diminuir a dor. O objetivo deste estudo foi analisar os efeitos de três doses de laser de baixa intensidade (808nm), na dor e edema imediatos, em ratos submetidos a lesão experimental do músculo gastrocnêmio lateral. Foram utilizados 24 ratos Wistar, distribuídos aleatoriamente em quatro grupos, sendo: GC - animais lesionados e tratados com aparelho desligado (simulacro); G20 - animais lesionados e irradiados com dose de 20J/ $\mathrm{cm}^{2} ; \mathrm{G} 50$ - animais lesionados e irradiados com 50J/cm; e G100 - animais lesionados e irradiados com $100 \mathrm{~J} / \mathrm{cm}^{2}$. Para a produção da lesão experimental, introduziu-se $0,1 \mathrm{~mL}$ de formalina a $5 \%$ no ventre do músculo gastrocnêmio lateral direito. A avaliação da dor ocorreu através do tempo de elevação da pata (TEP) e o edema foi avaliado com auxílio de paquímetro metálico; ambas as avaliações ocorreram pré-lesão, pós-lesão, pós-tratamento, duas, oito e 24 horas pós-lesão. Os resultados mostraram aumento significativo do TEP e da avaliação com paquímetro, após a indução da lesão. Conclui-se que laser nos parâmetros utilizados não produziu diminuição na dor e edema, em animais submetidos a lesão muscular com injeção de formalina a 5\%.
\end{abstract}

Palavras-chave: laser de daixa potência, analgesia, edema inflamatório.

\begin{abstract}
Low-level laser therapy induces to cellular bioestimulation and can accelerate wound healing, promote skeletal muscle regeneration, decrease inflammatory response, stimulate neovascularization and decrease pain. The aim of this study was to analyze the effects of three doses of low-level laser therapy (808 nm), in the immediate pain and edema, in rats submitted to experimental injury of the lateral gastrocnemius muscle. Twenty four Wistar rats, randomly distributed in the following four groups were used: CG - injured and treated with equipment turned off (sham); G20 - injured and treated with $20 \mathrm{~J} / \mathrm{cm}^{2} ; \mathrm{G} 50$ - injured and treated with $50 \mathrm{~J} / \mathrm{cm}^{2}$; and G100 - injured and treated with $100 \mathrm{~J} / \mathrm{cm}^{2}$. For the experimental injury production, $0.1 \mathrm{~mL}$ of formalin $5 \%$ was introduced in the right lateral gastrocnemius muscle girth. Pain was assessed through the Paw Elevation Time (PET), and edema was assessed with a metallic caliper. Both evaluations happened before injury, after injury, after treatment, 2, 8 and 24 hours after injury. The results showed significant increase of PET and of the caliper evaluation after injury induction. It has been concluded that the laser irradiation in the used parameters did not produce pain and edema decrease in rats submitted to muscular injury with formalin 5\% injection.
\end{abstract}

Keywords: low-level laser therapy, analgesia, inflammatory edema.

\section{INTRODUÇÃO}

Lesões musculares representam um dos traumas esportivos mais comuns que acometem atletas, adiando seu retorno à modalidade esportiva por semanas ou meses. A manifestação clínica, indicativa indireta da lesão, irá depender da gravidade e da natureza da mesma, podendo ser classificada em três categorias: a) leve (grau l): apresenta poucas fibras lesadas, com pequeno edema e desconforto, além de mínima perda de força e movimento; b) moderada (grau II): apresenta um número maior de fibras lesadas com perda de força, edema e desconforto mais intenso; c) grave (grau III): apresenta extensa lesão muscular, resultando em perda total da função do músculo(1).
No músculo lesado, a dor está associada à lesão estrutural e o edema, à destruição da integridade da membrana plasmática e da membrana basal(2). A dor é conceituada pela International Association for the Study of the Pain como "uma experiência sensorial e emocional desagradável associada com uma lesão tecidual presente ou potencial, descrita relativamente a essa lesão"(3). Resulta em incapacidade e inabilidade e tem como finalidade a proteção do organismo; permanecendo essa condição, pode resultar em atrofia muscular, hábitos de desuso e à consciente ou inconsciente proteção, levando à perda grave da função muscular(4).

O edema é definido como um tumor ou tumefação formado pelo extravasamento de elementos para o exterior dos vasos e a in- 
tensidade deste extravasamento estará correlacionada com o tipo e o grau da agressão tecidual(2).

As células musculares lesionadas não são substituídas por células novas. No sistema musculoesquelético são obtidos núcleos satélites adicionais, que se multiplicam e, dessa forma, se fundem com as fibras danificadas ${ }^{(5)}$. Essas células diferenciadas auxiliam na capacidade regenerativa do músculo esquelético por meio de mecanismos intrínsecos, restabelecendo a função contrátil(1).

A irradiação laser modula vários processos biológicos, como: aumento da respiração mitocondrial e síntese de ATP; acelera a cicatrização de feridas; promove a regeneração do músculo esquelético após lesão; diminui a resposta inflamatória e estimula a neoformação de vasos sanguíneos. A laserterapia também induz a síntese de proteínas regulatórias nas células satélites do músculo esquelético, por causa da ativação do ciclo celular ${ }^{(6)}$. A laserterapia de baixa intensidade clinicamente pode ser usada no tratamento de processos inflamatórios, bioestimulação celular ${ }^{(7)}$ e alívio da dor, devido ao seu efeito antiinflamatório e na melhora ou aceleração da regeneração tecidual ${ }^{(8)}$.

Visando estudar a capacidade reparatória do sistema musculoesquelético, pesquisadores vêm utilizando métodos de indução de lesão experimental como contusão(9), eletroestimulação e injeções de miotoxinas ${ }^{(5)}$.

O objetivo deste estudo consistiu em analisar os efeitos biomoduladores de três doses de irradiação $\left(20,50\right.$ e $\left.100 \mathrm{~J} / \mathrm{cm}^{2}\right)$ de laser de baixa intensidade $(808 \mathrm{~nm})$, na dor e edema imediatos, em ratos submetidos a lesão experimental do músculo gastrocnêmio lateral direito.

\section{MÉTODOS}

\section{Delineamento do estudo e amostra}

Foram utilizados 24 ratos machos, da linhagem Wistar, com $12 \pm$ 2 semanas de idade e 327,13 \pm 18,03 gramas de peso corporal médio, obtidos no Biotério Central da Universidade Estadual do Oeste do Paraná - Unioeste, e mantidos em biotério próprio do Laboratório do Grupo de Estudo das Lesões e Recursos Fisioterapêuticos. Os animais ficaram alojados em gaiolas de contenção de polipropileno, agrupados em número de três, com temperatura ambiente de $25^{\circ} \mathrm{C}$ e fotoperíodo de 12 horas claro/escuro controlados, recebendo água e ração ad libitum. O projeto foi conduzido segundo as Normas Internacionais de Ética em Experimentação Animal ${ }^{(10)}$.

\section{Grupos experimentais} sendo:

Os animais foram distribuídos aleatoriamente em quatro grupos,

- Grupo controle (GC, n=6): lesionados e não irradiados. Tratados com aparelho desligado.

- Grupo 20 (G20, n=6): lesionados e irradiados com laser de diodo (808nm) de baixa potência, com dose de $20 \mathrm{~J} / \mathrm{cm}^{2}$;

- Grupo 50 (G50, n=6): lesionados e irradiados com laser de diodo (808nm) de baixa potência, com dose de $50 \mathrm{~J} / \mathrm{cm}^{2}$;

- Grupo 100 (G100, n = 6): lesionados e irradiados com laser de diodo $(808 \mathrm{~nm})$ de baixa potência, com dose de $100 \mathrm{~J} / \mathrm{cm}^{2}$.

\section{Produção da lesão experimental}

Os animais foram sedados, previamente ao procedimento, com éter etílico. Após a tricotomia local, introduziu-se formalina a 5\% nos três grupos, em quantidade de $0,1 \mathrm{~mL}$, no ventre do músculo gastrocnêmio lateral. Para padronizar o local da lesão experimental, mediu-se $1 \mathrm{~cm}$ da interlinha articular posterior direita, com paquímetro metálico, marca Mitutoyo/Stainless-Hardened ${ }^{\star}$. A formalina foi introduzida através de injeção, com seringa para aplicação de insulina, com 12,7mm de comprimento e 0,33mm de calibre (30G).

\section{Teste de incapacidade}

O teste de incapacidade articular foi descrito em detalhes por Tonussi e Ferreira(11). Nesse teste, foram colocados ratos sobre um cilindro metálico com $30 \mathrm{~cm}$ de diâmetro realizando $3 \mathrm{rpm}$. O cilindro recoberto por uma tela metálica foi conectado a um dispositivo eletrônico e este a um computador, que averiguou o tempo total em que a pata lesionada permanecia em elevação (TEP - tempo de elevação da pata) durante um minuto, através de botas metálicas idênticas encaixadas nas patas posteriores do animal. Embora ambas as patas posteriores fossem calçadas, apenas a bota metálica direita repassava informações ao computador; a pata posterior esquerda permaneceu com a bota metálica apenas com a finalidade de transmitir ao animal as mesmas sensações do membro contralateral.

Segundo Bressan et al. ${ }^{(12)}$, animais que não sofreram nenhuma intervenção invasiva e não apresentam alterações de marcha demonstram TEP ao redor de 10 segundos; esse valor só aumenta quando o animal, com o membro lesionado, demonstra dor.

Os animais foram avaliados antes da lesão experimental, após a lesão (aproximadamente cinco minutos depois), após o tratamento e com dois, oito e 24 horas após a lesão.

\section{Avaliação do edema}

Para quantificar o edema na região da lesão experimental, foi utilizado paquímetro metálico, que foi precisamente posicionado na região posterior no terço médio do membro posterior do animal, a $1 \mathrm{~cm}$ de distância abaixo da fossa poplítea, utilizada como ponto de referência, realizando as medidas em sentido látero-medial. As coletas de dados foram realizadas nos períodos semelhantes aos da avaliação do TEP, sempre após tal avaliação.

\section{Tratamento da lesão experimental}

No tratamento da lesão experimental foi utilizado um laser modelo clínico portátil, marca DMC. A caneta utilizada apresentava comprimento de onda de 808nm, com potência de 100mW. Prévio ao início do experimento, o aparelho foi devidamente aferido para certificação da dose utilizada.

Para a aplicação da radiação laser de baixa intensidade no local da lesão experimental, os animais foram imobilizados em um contensor confeccionado de material termoplástico-PVC por ser atóxico e inerte, com um suporte de madeira, similar ao utilizado por Lirani ${ }^{(13)}$, deixando o membro de interesse exposto.

Para o tratamento, utilizou-se o método de aplicação transcutâneo pontual, em contato direto da caneta sobre a região da lesão em ângulo de $90^{\circ}$, de maneira semelhante à realizada por Amaral et al.(14). A terapia laser foi realizada imediatamente após a lesão experimental.

\section{Análise dos dados}

Os dados foram analisados com base na estatística descritiva (média, desvio-padrão) e inferencial com ANOVA medidas repetidas para análise intragrupos e ANOVA one-way para análise intergrupos; em ambos os casos foi utilizado o pós-teste de Tukey, sendo aceito o nível de significância de $p<0,05$.

\section{RESULTADOS}

A avaliação da presença da dor nos grupos GC, G20, G50 e G100, nos períodos pré-lesão, pós-lesão, pós-tratamento, duas horas, oito horas e 24 horas, observada pela deambulação através do TEP, mostrou aumento dos tempos, comparado com os valores pré-lesão; em nenhum caso houve diminuição do TEP ao comparar os valores pós-lesão com os momentos seguintes. Ao comparar entre os grupos, nos diferentes momentos de avaliação, não houve diferenças significativas (figura 1). 


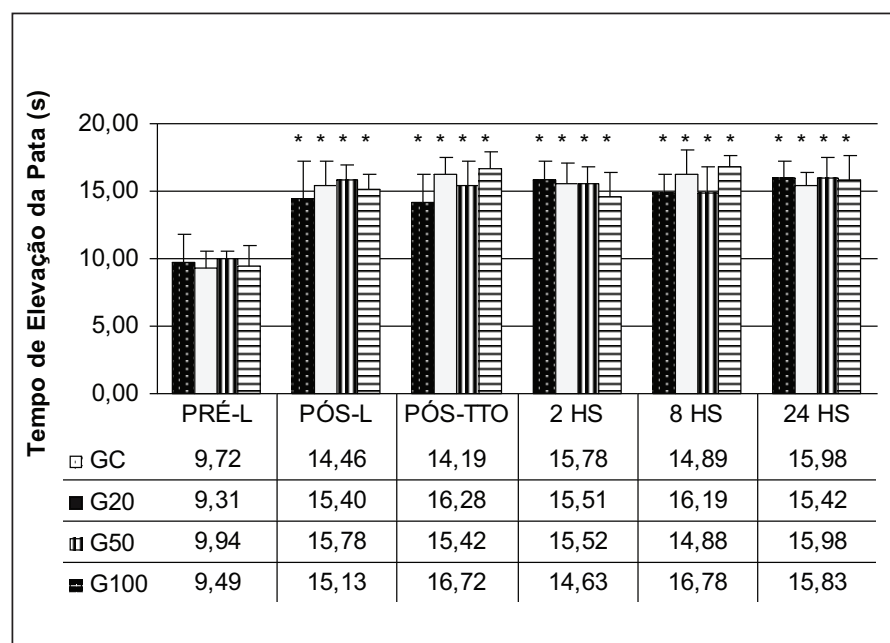

Figura 1. Avaliação da dor através dos valores do tempo de elevação da pata (TEP), nos momentos pré-lesão (PRÉ-L), pós-lesão (PÓS-L), pós-tratamento (PÓS-TTO), duas horas após a realização da lesão (2 HS), oito horas após a realização da lesão (8 HS) e 24 horas após a realização da lesão (24 HS); de acordo com o grupo: grupo controle (GC), grupo tratado com $20 \mathrm{Jcm}^{2}$ (G20), grupo tratado com $50 \mathrm{~J} / \mathrm{cm}^{2}$, grupo tratado com 100J/ $\mathrm{cm}^{2}$ (G100). * diferença estatisticamente significativa ao comparar com o valor pré-lesão.

A avaliação do edema, nos momentos semelhantes ao TEP mostrou aumento significativo do diâmetro médio-lateral, em todos os grupos, com exceção de GC na avaliação em 24 horas. De forma semelhante ao observado na avaliação do TEP, em nenhum grupo houve diminuição significativa do diâmetro muscular ao comparar os valores pós-lesão com os momentos seguintes. Mas, ao realizar a comparação entre os grupos, havia diferença significativa entre GC e G20 na avaliação prélesão, entre GC e G100 na avaliação pós-lesão, e entre GC e G100 na avaliação após 24 horas.

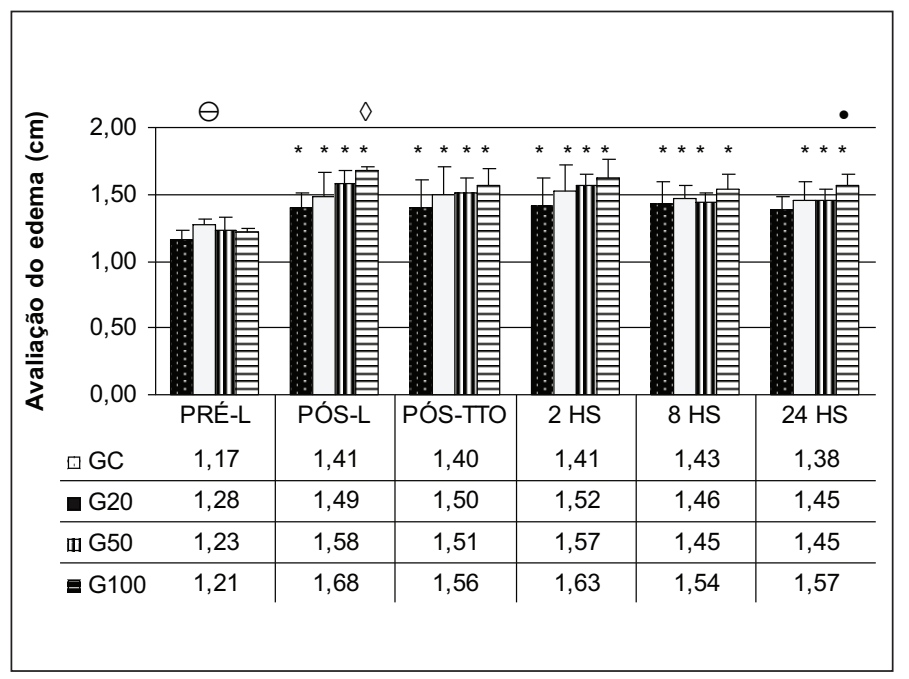

Figura 2. Avaliação do edema através do diâmetro médio-lateral da perna direita dos animais, na região dos gastrocnêmios, com uso de paquímetro metálico; nos momentos pré-lesão (PRÉ-L), pós-lesão (PÓS-L), pós-tratamento (PÓS-TTO), duas horas após a realização da lesão (2 HS), oito horas após a realização da lesão (8 HS) e 24 horas após a realização da lesão (24 HS); de acordo com o grupo: grupo controle (GC), grupo tratado com $20 \mathrm{Jcm}^{2}(\mathrm{G} 20)$, grupo tratado com $50 \mathrm{~J} / \mathrm{cm}^{2}$, grupo tratado com $100 \mathrm{~J} / \mathrm{cm}^{2}$ (G100). * diferença estatisticamente significativa ao comparar com o valor pré-lesão; $\ominus$ diferença estatisticamente significativa ao comparar GC com G20 no momento pré-lesão; $\searrow$ diferença estatisticamente significativa ao comparar GC com G100 no momento pós-lesão; • diferença estatisticamente significativa ao comparar GC com G100 após 24 horas de lesão.

\section{DISCUSSÃO}

O efeito da laserterapia de baixa intensidade no tratamento tecidual é controverso e sua eficácia para estimular a proliferação celular e promover o processo de regeneração é discutível na literatura. Porém, os efeitos atérmicos da irradiação laser sobre as células satélites pode alterar o processo de regeneração musculoesquelética em ratos através de efeitos trófico-regenerativos, analgésicos e antiinflamatórios ${ }^{(8,15,16)}$.

No presente estudo foi avaliada a presença de dor e de edema em ratos submetidos a lesão experimental, com formalina a 5\%, no músculo gastrocnêmio lateral direito.

Na análise da dor do membro posterior do animal, através do tempo de elevação, foi observada presença significativa da mesma em todos os grupos logo após a lesão experimental, indicando a produção da lesão em todos os grupos. O quadro álgico apresentado pelos animais permaneceu durante as primeiras 24 horas para os quatro grupos, não sendo observada diminuição da dor nos grupos tratados com laserterapia de baixa potência e no grupo controle. O que também foi observado em estudo prévio ${ }^{(17)}$, para o comprimento de onda de $670 \mathrm{~nm}$ e doses de 10 e $20 \mathrm{~J} / \mathrm{cm}^{2}$.

Esses resultados corroboram algumas pesquisas, como a realizada por Bingöl et al.(18), que utilizaram o laser de baixa intensidade AsGa (904nm) em pacientes com artralgia do ombro. Relataram melhora em alguns movimentos passivos e ativos e na sensibilidade à palpação, mas nenhuma melhora significativa na dor do grupo tratado comparado com o grupo controle.

Ainda, Oliveira et al.(16) avaliaram o efeito de diferentes doses (3 e $10 \mathrm{~J} / \mathrm{cm}^{2}$ ) de laser AsGa $(904 \mathrm{~nm})$ na regeneração do músculo tibial anterior de ratos. A irradiação foi repetida diariamente por cinco dias consecutivos, a primeira aplicação sendo feita duas horas após a indução da lesão experimental. Os autores não observaram diferenças entre os grupos tratados com $3 \mathrm{~J} / \mathrm{cm}^{2}, 10 \mathrm{~J} / \mathrm{cm}^{2}$ e o grupo placebo. Apenas observaram no grupo de $10 \mathrm{~J} / \mathrm{cm}^{2}$ aumento de peso corporal. Pogrel et al. ${ }^{(19)}$ avaliaram o efeito do laser AsGaAl (830nm) na proliferação, na adesão e na migração celular em cultura de fibroblastos. Não houve diferença entre o grupo tratado com terapia laser e o grupo não irradiado. Concluíram que o laser AsGaAl não demonstrou efeito bioestimulador em culturas de fibroblastos.

Por outro lado, há estudos que mostram o efeito positivo da laserterapia de baixa intensidade na regeneração do músculo tibial anterior em ratos induzidos à lesão por miotoxina ${ }^{(14)}$, no processo cicatricial em infarto crônico experimental(6) e na proliferação celular in vitro ${ }^{(20)}$

Na análise do edema do membro posterior direito do animal, foi observada presença significativa do mesmo em todos os grupos logo após a produção da lesão experimental. Para os grupos tratados, o aumento manteve-se significativo em todos os períodos de comparação, mas, para o grupo controle, não havia diferença significativa no edema após 24 horas da lesão.

Morrone et al.(21), utilizando um laser AsAlGa (780nm), com doses de $150 \mathrm{~J} / \mathrm{cm}^{2}, 250 \mathrm{~J} / \mathrm{cm}^{2}$ e $800 \mathrm{~J} / \mathrm{cm}^{2}$ sobre o músculo esquelético, após quatro dias de trauma, relataram diminuição no líquido dos espaços intersticiais e, conseqüentemente, diminuição de edema, após cinco e 10 dias de tratamento. 0 que difere do apresentado no presente estudo, mas, salienta-se que a avaliação ocorrida aqui foi no decorrer de 24 horas e as doses utilizadas para o tratamento foram inferiores às citadas acima.

A aplicação da radiação, logo após a realização da irritação química com a formalina, pode ter produzido aumento no extravasamento sanguíneo, pois, segundo Maegawa et al.(22), o laser de baixa potência produz vasodilatação arteriolar e aumento do fluxo sanguíneo, e, dessa forma, apesar da diminuição na produção de $P G E_{2}{ }^{(23)}$, a combinação da 
vasodilatação induzida pela lesão com a vasodilatação induzida pelo laser de baixa potência pode ter contribuído para maior formação do exsudato e, assim, não ter ocorrido a volta aos valores iniciais, como no grupo controle.

Salienta-se também a diferença observada entre o grupo controle com aquele tratado com $100 \mathrm{~J} / \mathrm{cm}^{2}$, a qual se mostrou significativa 24 horas após a lesão, podendo ter ocorrido devido à maior formação do edema com o tratamento de $100 \mathrm{~J} / \mathrm{cm}^{2}$. Porém, houve também tal diferença no momento pós-lesão, o que pode indicar falhas na avaliação ou, apesar de a lesão ser seriada, maior dano provocado nos animais do grupo $100 \mathrm{~J} / \mathrm{cm}^{2}$. Por isso, vale ressaltar como limitações deste estudo a ausência de visualização do tamanho e intensidade da lesão, bem como a não observação de possíveis alterações dos mediadores químicos envolvidos no processo inflamatório.

Por outro lado, Albertini et al. ${ }^{(24)}$ estudaram o laser AsAlGa com comprimento de onda de $650 \mathrm{~nm}$, comparando as doses de $1 \mathrm{~J} / \mathrm{cm}^{2}$, $2,5 \mathrm{~J} / \mathrm{cm}^{2}$ e $5 \mathrm{~J} / \mathrm{cm}^{2}$, em modelo inflamatório de edema em patas de ratos; demonstraram que o efeito antiinflamatório se inicia após a primeira aplicação de laser de baixa intensidade, e que a densidade de energia que obteve melhor efeito foi de $1 \mathrm{~J} / \mathrm{cm}^{2}$ e $2,5 \mathrm{~J} / \mathrm{cm}^{2}$, reduzindo o edema em $27 \%$ e 45,47\%, respectivamente.

Gonçalves e Parizotto(25) afirmam ser de grande importância a presença de um intervalo entre as aplicações, ou seja, a necessidade de continuidade da terapia, o que corrobora o relato de $\mathrm{Ng}$ et al. ${ }^{(26)}$ sobre a freqüência no uso da terapia, com doses cumulativas, ser superior ao uso de única aplicação.
Tais fatos relatados acima podem explicar a ausência de efeitos analgésicos e antiedematosos no presente estudo, visto que ocorreu apenas uma única aplicação da terapia laser. Além disso, foram aplicadas doses de 20, 50 e $100 \mathrm{~J} / \mathrm{cm}^{2}$, não sendo consideradas por Albertini et al.(27) e Honmura et al. ${ }^{(28)}$ doses ideais para obter efeitos da laserterapia de baixa intensidade. Mas, ressalta-se que na terapia com laser de baixa potência não há consenso, com relação a suas variáveis dosimétricas, como o comprimento de onda, densidade de energia e freqüência de aplicações. Visto que efeitos positivos de tal terapia são observados com doses baixas ${ }^{(27,28)}$, mas também com doses consideradas como terapeuticamente altas $(21,26,29)$.

\section{CONCLUSÃO}

Os efeitos imediatos da irradiação laser de baixa potência, $670 \mathrm{~nm}$, com 10 e $20 \mathrm{~J} / \mathrm{cm}^{2}$, não foram significativos para reduzir a dor e houve aumento no edema, em ratos submetidos a lesão experimental do músculo gastrocnêmio lateral direito com formalina a 5\%.

\section{AGRADECIMENTOS}

À Universidade Estadual do Oeste do Paraná (Unioeste) e ao Hospital Universitário do Oeste do Paraná (HUOP), pelo financiamento parcial deste estudo.

Todos os autores declararam não haver qualquer potencial conflito de interesses referente a este artigo.

\section{REFERÊNCIAS BIBLIOGRÁFICAS}

1. Järvinen $T A H$, Järvinen $T L N$, Kääriäinen $M$, Kalimo H, Järvinen M. Muscle injuries. Biology and treatment Am J Sports Med 2005;33:745-64.

2. Resende MA, Pereira LSM, Castro MSA. Proposta de um modelo teórico de intervenção fisioterapêutica no controle da dor e inflamação. Fisioterapia Brasil 2005;6:268-71.

3. Prentice WE. Modalidades terapêuticas em medicina esportiva. São Paulo: Manole, 2002.

4. Teixeira MJ, Marcon RM, Figueiró JAB. Dor, epidemiologia, fisiopatologia, avaliação, síndromes dolorosas e tratamento. São Paulo: Moreira Jr, 2001

5. Hill M, Wernick A, Goldspink G. Muscle satellite (stem) cell activation during local tissue injury and repair. J Anat 2003;203:89-99.

6. Oron U, Yaakobi T, Oron A, Mordechovitz D, Shofti R, Hayam G. Low-energy laser irradiation reduces formation of scar tissue after myocardial infarction in rats and dogs. Circulation 2001;103:296-301.

7. Greco M, Perlino E, Pastorea D, Guida G, Mama E, Quagliariellob E. Helium-neon laser irradiation of rat liver mitochondria gives rise to a new subpopulation of mitochondria: isolation and first biochemica characterization. J Photochem Photobiol B 1991;10:71-8.

8. Shibata Y, Ogura N, Yamashiro K, Takashiba S, Kondoh T, Miyazawa K, et al. Anti-inflammatory effect of linear polarized infrared irradiation on interleukin-1b-induced chemokine production in MH7A rheumatoid synovial cells. Lasers Med Sci 2005;20:109-13.

9. Minamoto VB, Bunho SR, Salvini TF. Regenerated rat skeletal muscle after periodic contusions. Braz J Med Biol Res 2001;34:1447-52.

10. Andersen ML, D'almeida V, Ko GM, Kawakami R, Martins PJF, Magalhães LE, et al. Princípios éticos e práticos do uso de animais de experimentação. São Paulo: UNIFESP, 2004

11. Tonussi CR, Ferreira SH. Rat knee-joint carrageenin incapacitation test: an objective screen for central and peripheral analgesics. Pain 1992;48:421-7.

12. Bressan E, Cunha FQ, Tonussi CR. Contribution of TNFa, IL-1b and CINC-1 for articular incapacitation, edema and cell migration in a model of LPS-induced reactive arthritis. Cytokine 2006;36:83-9.

13. Lirani APR. Estudo comparativo dos efeitos do ultrasom e do laser de baixa intensidade, no reparo ósseo de tíbia de ratos [Dissertação]. São Paulo: Universidade de São Paulo, 2004.

14. Amaral AC, Parizotto NA, Salvini TF. Dose-dependency of low energy HeNe Laser effect in regeneration of skeletal muscle in mice. Lasers Med Sci 2001;16:44-51.

15. Bibikova A, Oron U. Regeneration in denervated toad (Bufo viridis) gastrocnemius muscle and the promotion of the process by low energy laser irradiation. Anat Rec 1995;241:123-8.
16. Oliveira NM, Parizzotto NA, Salvini TF. GaAs (904-Nm) laser radiation does not affect muscle regeneration in mouse skeletal muscle. Lasers Surg Med 1999;25:13-21.

17. Borato E, Ciena AP, Bertolini GRF. Use of the laser InGaAIP, $670 \mathrm{~nm}$, in wistar rats muscular lesion. Fiep Bulletin 2008;78:50-3.

18. Bingöl Ü, Altan L, Yurtkuran M. Low-power laser treatment for shoulder pain. Photomed Laser Surg 2005;23:459-64.

19. Pogrel MA, Chen JW, Zhang K. Effects of low-energy gallium-aluminum-arsenide laser irradiation on cultured fibroblasts and keratinocytes. Lasers Surg Med 1997;20:426-32.

20. Pinheiro AL, Carneiro NS, Vieira AL, Brugnera A Jr, Zanin FA, Barros RA, et al. Effects of low-level laser therapy on malignant cells: in vitro study. J Clin Laser Med Surg 2002;20:23-6.

21. Morrone G, Guzzardella GA, Orienti L, Giavaresi G, Fini M, Rocca M, et al. Muscular trauma treated with a Ga-Al-As diode laser: in vivo experimental study. Lasers Med Sci 1998;13:293-8.

22. Maegawa Y, Itoh T, Hosokawa T, Yaegashi K, Nishi M. Effects of near-infrared low-level laser irradiation on microcirculation. Lasers Surg Med 2000;27:427-37.

23. Sakurai Y, Yamaguchi M, Abiko Y. Inhibitory effect of low lever laser irradiation on Ips stimuled protaglandin E2 production and cyclooxygenase-2 in human gingival fibroblasts. Eur J Oral Sci 2000;108:29-34.

24. Albertini R, Correia Fl, Ribeiro OW, Cogo JC, Antunes E, Teixeira S, et al. Análise do efeito do laser de baixa potência (AsGaAl) no modelo de inflamação de edema de patas em ratos. Fisioterapia Brasil 2002;3:5-15.

25. Gonçalves G, Parizotto NA. Fisiopatologia da reparação cutânea: atuação da fisioterapia. Rev Bras Fisiot 1998:21:5-13.

26. Ng GYF, Fung DTC, Leung MCP, Guo X. Comparison of single and multiple applications of GaAlAs laser on rat medial collateral ligament repair. Lasers Surg Med 2004;34:285-9.

27. Albertini R, Aimbire FS, Correa Fl, Ribeiro W, Cogo JC, Antunes E, et al. Effects of different protocol doses of low power gallium-aluminum-arsenate ( $\mathrm{Ga}-\mathrm{Al}-\mathrm{As}$ ) laser radiation $(650 \mathrm{~nm})$ on carrageenan induced rat paw edema. J Photochem Photobiol B 2004;74:101-7.

28. Honmura A, Yanese M, Obata J, Haruki E. Therapeutic effect of Ga-Al-As diode laser irradiation on experimentally induced inflammation in rats. Lasers Surg Med 1999;12:441-9.

29. Fung DTC, Ng GYF, Leung MCP, Tay DKC. Effects of a therapeutic laser on the ultrastructural morphology of repairing medial collateral ligament in a rat model. Lasers Surg Med 2003:32:286-93. 